\title{
Classification of brain lesions on magnetic resonance imaging using superpixel, PSO and convolutional neural network
}

\author{
Carolina L. S. Cipriano, Giovanni L. F. da Silva, Jonnison L. Ferreira, Aristófanes C. Silva, Anselmo Cardoso de Paiva \\ Núcleo de Computação Aplicada (NCA) \\ Universidade Federal do Maranhão (UFMA), São Luís - Brasil \\ Email: \{carol,giovannilucca,jonnison,ari,paiva\}@nca.ufma.br
}

\begin{abstract}
One of the most severe and common brain tumors is gliomas. Manual classification of injuries of this type is a laborious task in the clinical routine. Therefore, this work proposes an automatic method to classify lesions in the brain in 3D MR images based on superpixels, PSO algorithm and convolutional neural network. The proposed method obtained results for the complete, central and active regions, an accuracy of $87.88 \%, 70.51 \%, 80.08 \%$ and precision of $76 \%, 84 \%, 75 \%$ for the respective regions. The results demonstrate the difficulty of the network in the classification of the regions found in the lesions.
\end{abstract}

Index Terms-brain tumor classification, magnetic resonance imaging, convolutional neural networks, particle swarm optimization, superpixel

\section{INTRODUÇÃO}

Gliomas são tipos de tumores cerebrais que se originam das células gliais [1]. Eles são os principais tipos de tumores cerebrais, em torno de 40 a $60 \%$ das ocorrências [2]. São os tumores cerebrais mais comuns, com uma expectativa de vida de vários anos nos casos mais amenos ou uma expectativa de vida de no máximo 2 anos [3] nos casos mais agressivos.

O diagnóstico precoce de gliomas desempenha um papel importante na melhoria das possibilidades do tratamento [4]. Embora outras modalidades como Tomografia Computadorizada (TC), Tomografia por Emissão de Positrões (PET) sejam usadas em combinação para fornecerem mais informações sobre os tumores cerebrais, devido ao seu bom contraste dos tecidos moles e a ampla disponibilidade, a Ressonância Magnética (RM) é considerada a técnica padrão, fornecendo informações valiosas sobre a forma, tamanho e localização dos tumores cerebrais [5].

A tarefa de classificação manual da lesão, alguém de muito laboriosa devido ao grande volume de imagens geradas na rotina clínica, está sujeita a erros e divergências entre os especialistas. Não é tarefa fácil, pois as lesões apresentam formas e tamanhos diversos. Uma classificação automática das regiões (completa, central e ativa) forneceria ao especialista uma segunda opinião, auxiliando no seu trabalho.

Diante disso, este trabalho propõe um método automático para a classificação de lesões no cérebro em imagens de RM 3D baseado em superpixels, algoritmo de otimização de enxame de partículas, do inglês Particle Swarm Optimization
(PSO), e a rede neural convolucional, do inglês Convolutional Neural network (CNN). Como contribuição desse trabalho, ressalta-se o uso do algoritmo de otimização de enxame de partículas para otimização das técnicas de superpixels e seus respectivos parâmetros para uma melhor classificação. Contribui também para o enriquecimento dos estudos sobre classificação de gliomas, com finalidade de auxiliar o especialista no diagnóstico precoce de lesões. Além disso, este trabalho desenvolve um modelo de treinamento de redes convolucionais para classificação de gliomas.

$\mathrm{Na}$ literatura existem poucos trabalhos relacionados para a classificação de lesões em imagens de RM. Mas há duas principais abordagens sobre as regiões de interesse. A primeira abordagem trabalha com todas as sub-regiões da lesão (necrose, edema, núcleo sólido e núcleo). A segunda abordagem trabalha com 3 grandes regiões: completa (necrose, edema, núcleo sólido e núcleo), central (necrose, núcleo sólido e núcleo) e ativa (núcleo).

$\mathrm{Na}$ abordagem por grandes regiões, [6] propuseram um método de segmentação automática baseado em redes neurais convolucionais e extração de patches.

O trabalho proposto por 7 utilizou um modelo discriminativo para detecção de tumores a partir de imagens de RM. A parte principal do modelo é construída em torno do classificador de floresta aleatória, do inglês Random Forest (RF).

Este artigo está organizado da seguinte forma: A Seção II apresenta os materiais e métodos para o trabalho proposto. A Seção III apresenta e discute os resultados. Finalmente, a Seção IV apresenta as considerações finais deste estudo.

\section{MATERiAis E MÉtodos}

A metodologia proposta é dividida em quatro etapas, como ilustrado na Figura 1. Em resumo, a primeira etapa detalha os materiais usados como as imagens de RM. A segunda etapa consiste na aplicação das técnicas de melhoramento de imagens. Apos esta etapa, a classificação é realizada através da técnica de superpixels, o algoritmo de otimização de enxame de partículas e a rede neural convolucional. Finalmente, os resultados são avaliados. 


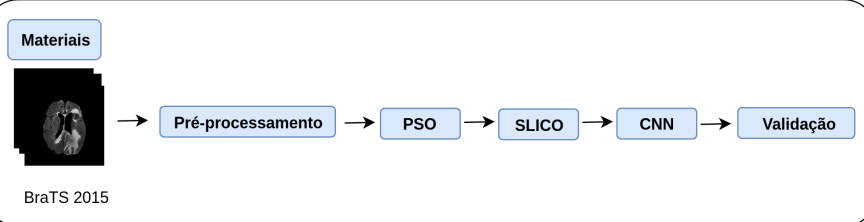

Fig. 1. Etapas do método proposto.

\section{A. Materiais}

A base de dados usada no trabalho é a Multimodal Brain Tumor Image Segmentation Benchmark (BrasTS) [8] composta por pacientes contendo gliomas de alto e baixo grau. A marcação dos tumores foi realizada de forma manual por diversos especialistas e todos os pacientes compartilham de quatro modos de ressonância: T1, T1c, T2 e FLAIR [9], como mostrado na Figura 2. Cada paciente possui um total de 155 fatias. Todas as imagens foram removidas do crânio para garantir anonimização dos pacientes. Os gliomas de alto grau são mais agressivos e infiltrativos, por esse motivo foram escolhidos para este trabalho. No total, a base contém 220 pacientes contendo gliomas de alto grau.

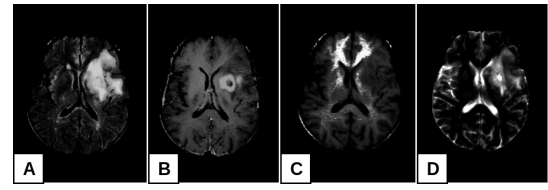

Fig. 2. (A) Flair, (B) T1, (C) T1c e (D) T2.

Além da marcação dos tumores cerebrais, a base disponibiliza a classificação da lesão em quatro regiões intra-tumorais, a saber: necrose, edema, núcleo sólido e núcleo. Para avaliar o desempenho dos algoritmos de classificação e segmentação, no entanto, a maioria dos trabalhos agrupam os diferentes estruturas em três regiões tumorais mutuamente inclusivas que melhor representam as tarefas de aplicação clínica. A região completa do tumor (incluindo todas as quatro estruturas tumorais). A região central do tumor (incluindo todas as estruturas tumorais, exceto o edema) e a região ativa do tumor (contendo apenas a região do núcleo). Como mostrado na Figura 3.

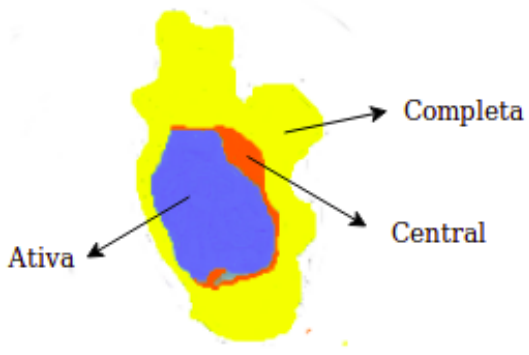

Fig. 3. Imagem com cores ilustrativas de marcações de regiões intra-tumorais.

\section{B. Pré-processamento}

Duas técnicas de pré-processamento foram realizadas para normalizar os valores de intensidade das imagens de RM nas quatro modalidades. As imagens foram obtidas de diferentes equipamentos, os tecidos do cérebro podem apresentar intensidades muito diferentes em diferentes exames. Para reduzir esse tipo de problema inter-exame, a especificação do histograma [10] é comumente aplicada. O segundo passo consiste na normalização das intensidades intra-exame, como mostrado na Equação 1, com o objetivo de tornar a média próxima de zero e o desvio padrão menor [6]. A Figura 4 mostra um exemplo.

$$
\text { pixel }=\frac{\text { pixel }- \text { Média(fatia) }}{\text { Desvio Padrão(fatia) }}
$$

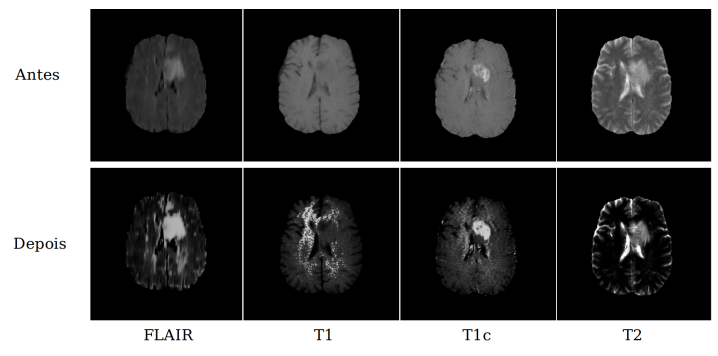

Fig. 4. Fatias antes e depois do pré-processamento.

\section{Extração dos superpixels baseado no algoritmo PSO}

A técnica de superpixel reduz significativamente o custo de memória, enquanto potencialmente aumenta a precisão de detecção. As técnicas de superpixels analisadas foram o agrupamento iterativo linear simples (SLIC) [11], e a sua variação SLIC otimizada (SLICO) [12].

As duas técnicas de superpixels citadas (SLIC e SLICO) possuem dois parâmetros a serem inicializados. Tamanho do superpixel médio e fator de compactação. A escolha de qual algoritmo de agrupamento (SLIC e SLICO), parâmetros (tamanho médio do superpixel e o fator de compactação) e o modo da imagem de RM (FLAIR, T1, T1c, T2) que serão aplicados implicam diretamente no desempenho da classificação da lesão, pois cada região da lesão pode estar melhor representada em um modo de RM. Portanto, a fim de otimizar tais parâmetros, o algoritmo de otimização de enxame de partículas (PSO) foi usado.

O algoritmo PSO [13] é uma técnica evolutiva inspirada no comportamento colaborativo de populações biológicas. O PSO pode gerar uma solução de alta qualidade dentro de um tempo de execução mais curto e exibindo uma característica de convergência estável mais eficaz do que outras técnicas de otimização [14]. Foi utilizado para buscar os melhores parâmetros de forma automática.

Os parâmetros a serem otimizados pelo PSO são apresentados na Figura 5. O modo da imagem de RM pode ser do tipo FLAIR, T1, T1c e T2, a técnica de superpixel pode ser o SLIC ou o SLICO, o tamanho do superpixel pode variar de 4 a 10 , e por fim, o fator de compactação pode variar de 0 
a 10. Tais intervalos de valores foram definidos baseados nas propriedades das imagens de RM do cérebro. Portanto, cada partícula é definida por uma lista de quatro valores, cada uma definida pelo seu intervalo.

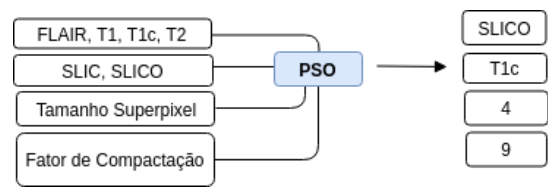

Fig. 5. Parâmetros a serem otimizados pelo PSO

Após a definição dos parâmetros e seus intervalos, é definido um enxame inicial com 10 partículas, sendo 9 partículas iniciadas de maneira aleatória e 1 partícula iniciada manualmente, considerada como uma boa partícula, que foi definida empiricamente com base nos primeiros testes com o PSO, onde todas as 10 partículas eram iniciadas aleatoriamente. Para a avaliação da função objetivo foi usado o coeficiente dice [15] entre a segmentação da lesão com tais parâmetros e a marcação dos especialistas, usando 10 pacientes, também escolhidos aleatoriamente.

\section{Classificação dos superpixel usando CNN}

As CNNs são o modelos de Deep Learning muito conhecidas e largamente utilizadas para o processamento de imagens [16]. São compostas por camadas convolucionais que processam as entradas considerando campos receptivos locais.

Serão usadas três CNNs para a classificação das regiões, uma para cada região da lesão (completa, central e ativa), como mostrado na Figura 6. A arquitetura da CNN usada no trabalho, está descrita na Tabela I . A arquitetura da rede foi inspirada na literatura, porém modificada e ajustada de forma empírica com base em repetitivos testes para chegar a uma melhor classificação do problema a ser solucionado.

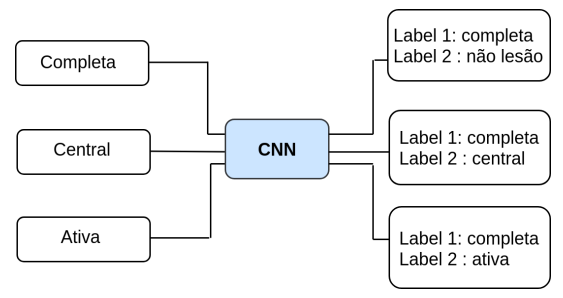

Fig. 6. Treinamento das regiões

TABLE I

ARQUiTETURA DA CNN.

\begin{tabular}{|c|c|}
\hline Entrada & 10x10x4 \\
\hline Convolução 1 & 64 filtros + Leaky ReLU (alfa 0.2) \\
\hline Dropout & 0.25 \\
\hline Convolução 2 & 128 filtros + Leaky ReLU (alfa 0.2) \\
\hline Dropout & 0.25 \\
\hline Completamente Conectada & 512 + Tangente Hiperbólica \\
\hline Saída & 2 neurônios + Softmax \\
\hline
\end{tabular}

Uma vez que a CNN é uma técnica de aprendizado de máquina supervisionado, é necessário rotular cada um dos superpixels extraídos. Para isso, utilizou-se a marcação dos especialistas. A melhor partícula encontrada com o PSO foi a partícula [ 2, 1, 4, 9], ou seja, usaremos o modo de RM T1c, o algoritmo de agrupamento SLICO, o tamanho médio do superpixel será 4 e seu fator de compactação 9. Após a aplicação do algotimo na imagem, faz-se necessário fazer a intersecção com o ground truth com o propósito de saber qual a classe predominante daquele superpixel. Depois, é retirada a mesma região do superpixel no modo T1c para os demais modos (Flair, T1, T2), empilhando-os em uma estrutura 10x10x4 que será a entrada da rede, como mostrado na Figura 7.

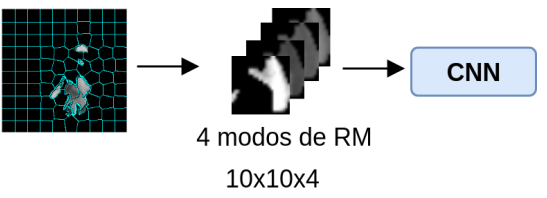

Fig. 7. Entrada da rede

A quantidade de rótulos geradas para a região completa foram 1.968.232, onde 236.603 pertencem a classe lesão e 1.731 .629 pertencem ao tecido saudável. Para a central, o total de rótulos gerados foram de 220.416, onde 72.577 pertencem a classe central e 147.839 pertencem ao restante da lesão. Para a região ativa, o total de rótulos gerados foram 220.416 , onde 42.687 pertencem a classe ativa e 177.729 ao restante da lesão.

\section{Resultados E Discuss Ão}

Esta seção apresenta e discute os resultados obtidos com o método proposto para a classificação de lesões no cérebro em imagens de RM 3D. A estratégia para análise dos resultados é detalhada como segue: 1) divisão da base de dados, 2) otimização da técnica de superpixel usando o algoritmo PSO, e 3) classificação dos superpixels.

\section{A. Divisão da base de dados}

A base de dados da BrasTS, conforme descrita na Seção II-A, contém 220 exames de RM, juntamente com a marcação dos especialistas. Para avaliar o método proposto foram usados apenas 100 exames da base por conta da limitação de hardware. Então, a base de dados foi dividida aleatoriamente em três conjuntos: treinamento, validação e teste. $\mathrm{O}$ conjunto de treinamento contém 74 exames de RM, o conjunto de validação contém 13 exames de RM e o conjunto de testes contém 13 exames de RM.

\section{B. Otimização da técnica de superpixel usando o PSO}

A busca do PSO foi definida para todas as classes da lesão e não somente para suas regiões. São 4 classes e 3 regiões. A região completa é definida por todas as classes (necrose, edema, núcleo sólido e núcleo), a classe central é formada por (necrose, núcleo sólido e núcleo) e por ultimo, a classe ativa é formada somente pelo núcleo.

Os resultados dos dices obtidos pelo algoritmo PSO nos exames do conjunto de 10 pacientes, foram: para a classe 
necrose, a melhor partícula foi definida como T1, SLICO, 4, 4; na classe edema, a melhor partícula encontrada foi T1c, SLICO, 4, 9; para a classe núcleo sólido, a melhor partícula foi definida como T1c, SLICO, 4, 5, e por fim, na classe núcleo a melhor partícula encontrada foi T1c, SLICO, 4, 5. Após a otimização de forma individual, a partícula escolhida para as quatro sub-regiões foi T1c, SLICO, 4, 9. Essa partícula obteve a maior média de dice e o menor desvio padrão quando aplicada em todas as regiões. Logo, a mesma representaria melhor todas as regiões.

\section{Classificação dos superpixels}

A configuração do treinamento de cada CNN foi definido para 50 épocas com o tamanho do batch igual a 32 superpixels e a função de otimização Adam com a taxa de aprendizado de 0,0002 .

O objetivo de treinar cada região com redes neurais convolucionais separadamente é diminiuir o número de falsos negativos, uma vez que cada rede tenta discriminar entre a região de interesse e todo o restante da lesão, tornando um uma classificação de três regiões em 3 problemas binários.

Serão usadas quatro métricas a fim de avaliar o modelo. A primeira é a acurácia, em problemas de classificação é o número de previsões corretas feitas pelo modelo sobre todas as previsões de tipos feitas. Mas como essa métrica não confiável para problemas com classes desbalanceadas, outras métricas serão usadas. A segunda é a precisão, uma medida que nos diz qual a proporção de rótulos que diagnosticamos como determinada região, na verdade, são daquela região. A terceira é o recall, onde o modelo deve de qualquer maneira encontrar todos os rótulos da região de interesse, mesmo que classifique alguns rótulos da lesão como rótulos da região específica. $\mathrm{O}$ f1-score, é simplesmente observar somente uma métrica ao invés de duas precisão e recall, pois é um indicativo de que ou a precisão ou o recall está baixo.

Todas as regiões estão desbalanceadas, o que afeta diretamente no resultado das redes. Para os dados de treinamento, a região completa representa somente $12,02 \%$ dos rótulos da imagem completa; a região central representa somente 32,93 \% dos rótulos de lesão e, por fim, a região ativa representa somente 19,37 \% dos rótulos de lesão. A Figura 8 demonstra essa proporção.

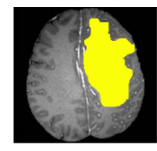

Completa

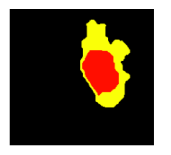

Central

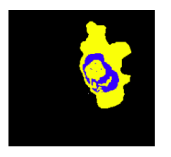

Ativa
Fig. 8. Proporções da região completa, central e ativa habitualmente encontradas nas fatias. Cores ilustrativas.

Os primeiros treinamentos para as regiões obtiveram acurácia de 76,71 \%,71,50\%,73,05\% para as regiões completa, central e ativa, respectivamente. Diante do desbalanceamento dos dados optou-se por usar o balanceamento dos pesos para cada treinamento e sua respectiva região, com a proporção da classe com menos instancias sobre a classe maior instancias. $\mathrm{O}$ resultado das dos treinamentos com o balanceamento dos pesos está nas Tabelas II, III e IV.

TABLE II

RESUlTADOS DA REDE PARA LESÃO COMPLETA.

\begin{tabular}{|l|l|}
\hline & Completa \\
\hline Acurácia & 0,87 \\
\hline Precisão & 0,76 \\
\hline Recall & 0,88 \\
\hline F1-score & 0,81 \\
\hline
\end{tabular}

TABLE III

RESUlTADOS DA REDE PARA LESÃO CENTRAL.

\begin{tabular}{|l|l|}
\hline & Central \\
\hline Acurácia & 0,70 \\
\hline Precisão & 0,84 \\
\hline Recall & 0,71 \\
\hline F1-score & 0,77 \\
\hline
\end{tabular}

TABLE IV

RESUlTADOS DA REDE PARA LESÃO ATIVA.

\begin{tabular}{|l|l|}
\hline & Ativa \\
\hline Acurácia & 0,80 \\
\hline Precisão & 0,75 \\
\hline Recall & 0,80 \\
\hline F1-score & 0,77 \\
\hline
\end{tabular}

Outro ponto, é tamanho da entrada da rede. Ele tem grande impacto na classificação da rede. Uma vez que o tamanho da região média do superpixel é 4 , definiu-se empiricamente o tamanho de entrada como 10x10 onde o fundo é a própria imagem. Definir um tamanho de imagem de entrada muito pequena, faz com que a rede tenha menos informação para discriminar, além de aumentar o número de rótulos. Por outro lado, aumentar o tamanho, faz a rede ter um desempenho melhor da classificação nas regiões mais bem delimitadas e mais representadas, como a região completa.

Para a região completa que é região com maior desbalanceamento o resultado melhorou em mais de $10 \%$, essa região é a que tem o melhor desempenho da rede, pois possui os tecidos mais contrastantes e fáceis de serem aprendidos pela rede (a lesão completa e a região saudável). A região central teve uma pequena queda no desempenho e é a mais difícil. Por último, a região ativa melhorou consideravelmente.

\section{CONCLUSÃO}

Os tumores cerebrais são problemas graves, independente do seu diagnóstico. A detecção precoce é de extrema importância para a sobrevida do paciente. Por isso, métodos assistidos por computador são comumente desenvolvidos e a análise automática de lesões auxiliam de forma expressiva os especialistas.

Os resultados da classificação dos superpixels foram promissores, obtendo uma acurácia média $87.88 \%, 70.51 \%$, $80.08 \%$ e precisão de $76 \%, 84 \%, 75 \%$ para as respectivas regiões. É importante destacar que esse trabalho contou com 
apenas 100 exames de uma base de 220 exames disponíveis para gliomas alto grau.

Como trabalho futuro, nós identificamos alguns pontos que podem ser aprimorados a fim de obter resultados melhores. $\mathrm{O}$ primeiro consiste em técnicas de sobre-amostragem para o balanceamento dos dados e comparação com o balanceamento dos pesos, o segundo consiste em incluir outras técnicas de superpixels na etapa de otimização. Por ultimo, pretende-se segmentar as regiões classificadas.

\section{REFERENCES}

[1] A. Omuro and L. M. DeAngelis, "Glioblastoma and other malignant gliomas: a clinical review," Jama, vol. 310, no. 17, pp. 1842-1850, 2013.

[2] M. L. Goodenberger and R. B. Jenkins, "Genetics of adult glioma," Cancer genetics, vol. 205, no. 12, pp. 613-621, 2012.

[3] M. Havaei, A. Davy, D. Warde-Farley, A. Biard, A. Courville, Y. Bengio, C. Pal, P.-M. Jodoin, and H. Larochelle, "Brain tumor segmentation with deep neural networks," Medical image analysis, vol. 35, pp. 18-31, 2017.

[4] B. Stewart, C. P. Wild et al., "World cancer report 2014," 2014.

[5] A. Işın, C. Direkoğlu, and M. Şah, "Review of mri-based brain tumor image segmentation using deep learning methods," Procedia Computer Science, vol. 102, pp. 317-324, 2016.

[6] S. Pereira, A. Pinto, V. Alves, and C. A. Silva, "Brain tumor segmentation using convolutional neural networks in mri images," IEEE transactions on medical imaging, vol. 35, no. 5, pp. 1240-1251, 2016.

[7] L. Lefkovits, S. Lefkovits, and M.-F. Vaida, "Brain tumor segmentation based on random forest," Memoirs of the Scientific Sections of the Romanian Academy, vol. 39, no. 1, pp. 83-93, 2016.

[8] B. H. Menze, A. Jakab, S. Bauer, J. Kalpathy-Cramer, K. Farahani, J. Kirby, Y. Burren, N. Porz, J. Slotboom, R. Wiest et al., "The multimodal brain tumor image segmentation benchmark (brats)," IEEE transactions on medical imaging, vol. 34, no. 10, p. 1993, 2015.

[9] UCSF, "Magnetic resonance imaging (mri)," https://radiology.ucsf.edu/patient-care/services/mriaccordion-whatare-the-risks-of-contrast, 2019, accessed: 2019-07-01.

[10] R. C. Gonzalez and R. C. Woods, Processamento digital de imagens. Pearson Prentice Hall, 2010.

[11] R. Achanta, A. Shaji, K. Smith, A. Lucchi, P. Fua, S. Süsstrunk et al., "Slic superpixels compared to state-of-the-art superpixel methods," IEEE transactions on pattern analysis and machine intelligence, vol. 34, no. 11, pp. 2274-2282, 2012.

[12] P. H. B. Diniz, T. L. Azevedo Valente, J. O. Bandeira Diniz, A. C. Silva, M. Gattass, N. Ventura, B. C. Muniz, and E. L. Gasparetto, "Detection of white matter lesion regions in mri using slico and convolutional neural network," Computer methods and programs in biomedicine, vol. 167, pp. 49-63, 2018.

[13] R. Eberhart and J. Kennedy, "A new optimizer using particle swarm theory," in Micro Machine and Human Science, 1995. MHS'95., Proceedings of the Sixth International Symposium on. IEEE, 1995, pp. $39-43$.

[14] G. L. F. d. Silva, T. L. A. Valente, A. C. Silva, A. C. de Paiva, and M. Gattass, "Convolutional neural network-based pso for lung nodule false positive reduction on ct images," Computer methods and programs in biomedicine, vol. 162, pp. 109-118, 2018.

[15] L. R. Dice, "Measures of the amount of ecologic association between species," Ecology, vol. 26, no. 3, pp. 297-302, 1945.

[16] M. A. Ponti and G. B. P. da Costa, "Como funciona o deep learning," arXiv preprint arXiv:1806.07908, 2018. 